\title{
Memoria e conoscenza. Il castello di Belmonte in Calabria
}

\author{
Memory and knowledge. The castle of Belmonte in Calabria
}

\author{
Caterina Gattuso $^{a}$, Marco Castriota ${ }^{b}$, Philomène Gattuso ${ }^{c}$, Francesca Saggio $^{d}$ \\ Università della Calabria, Rende (CS), Italy \\ a caterina.gattuso@unical.it; ${ }^{\mathrm{b}}$ marco.castriota@fis.unical.it; ${ }^{\mathrm{c}}$ philomene.gattuso@ unical.it; ${ }^{\mathrm{d}}$ francescasaggio@live.com
}

\begin{abstract}
A small A small village located in Italy on the Calabrian Tyrrhenian coast, Belmonte Calabro has its historic center with a typical medieval urban structure that has remained almost unchanged over the centuries and is characterized by the presence of the ruins of a castle and its surrounding environments whose. The planimetry succeeds to be identified because it is bordered by a wall, only partially preserved, pronounced by towers and marked by a road that, in its main points still existing, follows its development. The castle, built on the hill's top of a tuff nature, in an elevated position respect to the urban core, had a plan with a roughly quadrangular shape with four imposing square towers. Of particular note is its curtain wall that originally had four doors, which opened in correspondence at the four cardinal points. In addition to having suffered several collapses in many parts of its structure due to the various earthquakes that occurred over time, as well as various looting and the siege by the French artillery dating back to the early 1800 s, the castle is currently subjected to degrading actions due to the attack of biological type, which manifests itself with a widespread presence of patinas, as well as those due to a thick weed vegetation that affects many of the surfaces of its structure.

The study aims to provide a useful contribution to reconstructing the profile of the original structure of the ancient castle. To obtain, therefore, more information about it, a specific survey plan was developed to characterize its constituent materials and also its state of preservation. To this end, in correspondence of structural parts still intact, samples were collected that were analyzed and characterized in the laboratory by Raman Spectroscopy.
\end{abstract}

Keywords: Castle, Belmonte, materials, Raman.

\section{Introduzione}

Le antiche cinte murarie connotano in modo rilevante il nucleo urbano cui appartengono, esse infatti, ne sono parte integrante e qualificante.

In genere circondavano l'abitato con l'obiettivo di creare una barriera nei confronti degli attacchi provenienti dall'esterno.

A Belmonte Calabro, piccolo comune italiano della provincia di Cosenza, in Calabria le mura furono costruite come opera di difesa del castello e del suo nucleo abitato, che erano collocati in cima ad una altura dalla quale si poteva avere

un'ampia visuale che consentiva il controllo di un vasto tratto di mar Tirreno.

Attualmente nonostante il nucleo fortificato presenti un notevole stato di degrado a causa dei danni che le sue strutture e i suoi materiali costitutivi hanno dovuto subire nel tempo, sono ancora visibili e riconoscibili varie parti della sua conformazione originaria.

Con il presente studio si vuole fare una indagine conoscitiva con lo scopo di fornire un contributo volto a sistematizzare le informazioni esistenti 

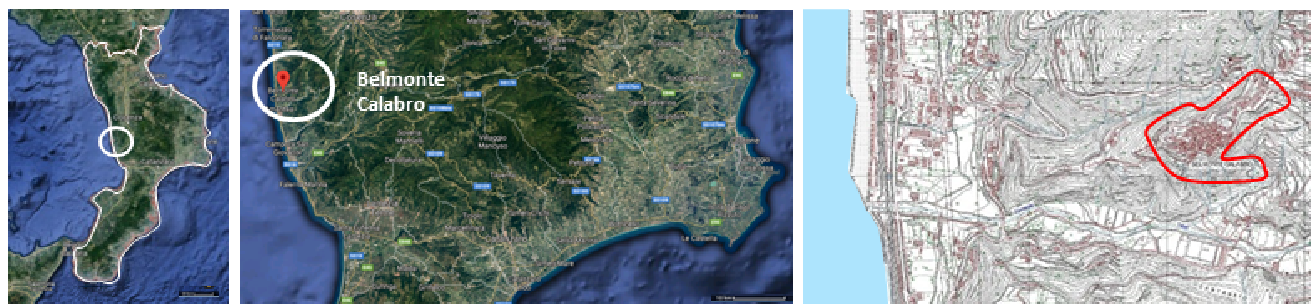

Fig. 1. Il contesto territoriale.

che lo riguardano e di acquisirne inoltre delle nuove in modo da delineare in maniera più efficace il suo quadro conoscitivo.

Data la complessità e la rilevante diversità di dati e di informazioni che occorre raccogliere e elaborare per raggiungere tali obiettivi si rende necessario organizzare e coordinare attraverso azioni di confronto e di sinergie i contributi provenienti da ambiti disciplinari diversi contemplando nello stesso tempo una adeguata integrazione transdisciplinare finalizzata al superamento delle specificità così da consentire una visione estesa e completa della situazione.

È sulla base di tali considerazioni che è stato affrontato e svolto lo studio riguardante il sistema delle mura difensive e il castello di Belmonte Calabro.

\section{Il contesto territoriale ed urbano}

Il centro di Belmonte (Bellimontum in latino, Bellimunti in dialetto locale) è posto su un terrazzo naturale a $220 \mathrm{mt}$ sul livello del mare. Il suo castello di origini normanne conserva, in parte, le antiche mura di cinta in origine scandite da tre porte: la Porta di Mare, che era affiancata da una torre; la Porta di Terra, posta ai piedi del castello; la Porta Laterale, ancora visibile, che si apre a nord verso il Vallone cui è rivolta. In origine, varcato l'ingresso del muro di cinta, vi era uno spiazzo a cielo aperto detto "vaglio del castello". Da esso si giungeva al corpo di guardia, ai magazzini e alla scuderia.Nel territorio dove venne costruito il castello, al tempo dei Normanni, nel secolo IX, vi erano tanti piccoli villaggi appartenenti all'antica città di Amantea i cui abitanti per difendersi dagli attaccati dei saraceni, al loro ritiro nel secolo XI, pensarono di fortificare la loro posizione edificando sulla col- lina rocciosa una prima torre rotonda e in seguito nel XII secolo alla fine del secolo successivo altre due che vennero collegate tra loro e trasformate in cittadella.

$\grave{E}$ in tale contesto territoriale che venne costruito il complesso fortificato che venne ampliato poi in una fase successiva con l'edificazione della cinta muraria (Gattuso, 2011).

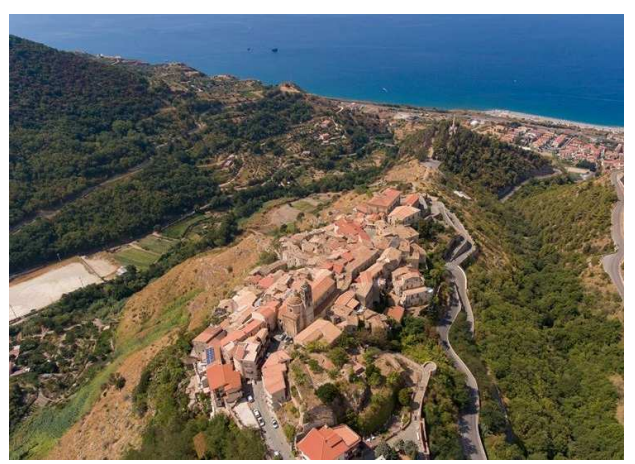

Fig. 2. Vista aerea del centro storico.

\section{Il contesto storico}

Secondo alcuni storici il toponimo del paese, Belmonte, si deve al Maresciallo del Regno Drogone di Beaumont che nel 1270-1271 realizzò il castello per ordine dei d'Angiò di Francia, altri ritengono invece che sia da rapportare all'amenità del colle sul quale sorse poi il paese.

Comunque attorno al castello cominciò già da subito a formarsi un nucleo di abitazioni che diedero origine nel 1280 ad un vero e proprio villaggio denominato San Bonaventura. La pianta del castello a quel tempo presentava una forma piuttosto quadrangolare con ai lati quattro torri di base quadrata. Il muro di cinta che venne edificato dai conti Salvacossa negli anni ' 80 del 


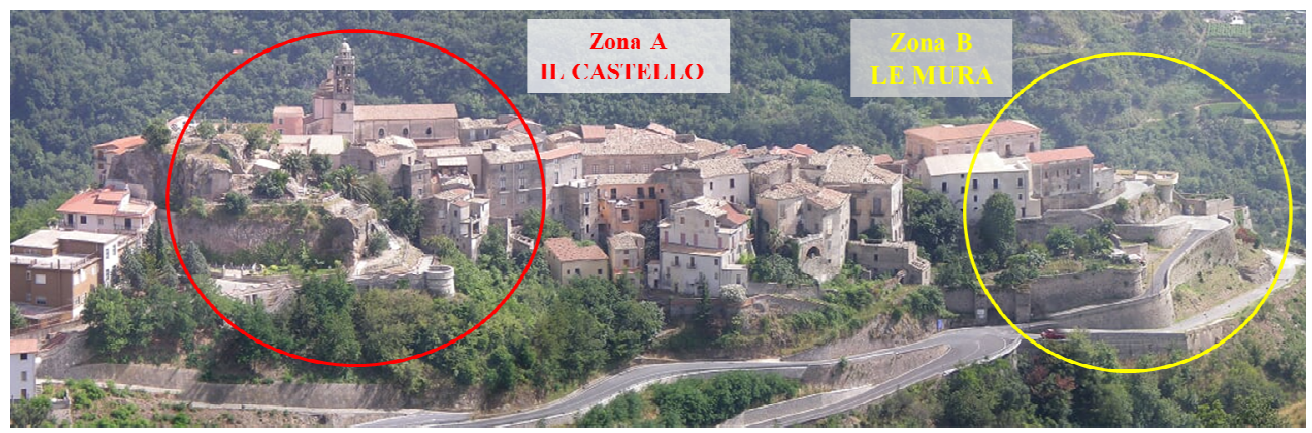

Fig. 3. Vista panoramica del nucleo storico, dei resti del castello e delle sue mura.

1700, secondo le fonti, presentava quattro porte, che si aprivano sui quattro punti cardinali. Quelle poste ad ovest e ad est, erano rivolte verso la valle del fiume Verrei, un'altra situata a nord, che dominava il vallone della porta, sulla cosiddetta fossa di Cardella, mentre la quarta collocata a sud, si apriva verso l'abitato.

Fu con il terremoto del 1638, che si ebbero i primi rilevanti danni, alcuni ambienti infatti divennero inabitabili e resi accessibili solo dopo essere stati effettuati interventi di restauro. Più rilevanti furono i danni causati dal terremoto del 1783 , che provocò il crollò il tetto. Con l'assedio dei francesi del 1806-1807 il castello, venne quasi completamente distrutto (Valenti, 1973). Sono rimasti solo dei ruderi superstiti ancora visibili nonostante le demolizioni delle parti pericolanti effettuate dai francesi e poi dal genio militare a seguito dei danni provocati dal sisma del 1905. Attualmente in esso si trova la biblioteca comunale ed un'area pic-nic panoramica.

\section{Il castello e le mura}

Il complesso fortificato antico, può considerarsi composto da due zone principali quella A relativa al Castello e la B riguardante le mura.

Per descrivere più dettagliatamente i ruderi del castello e del centro storico, la zona A è stata a sua volta ripartita in cinque aree (Gattuso, 2012).

Durante i sopralluoghi effettuati nella zona A è emerso che nell' area 1 esistono ancora delle parti originarie di ciò che rimane del castello, nonostante essa sia è stata soggetta negli anni a ristrutturazioni e rifacimenti degli esterni.

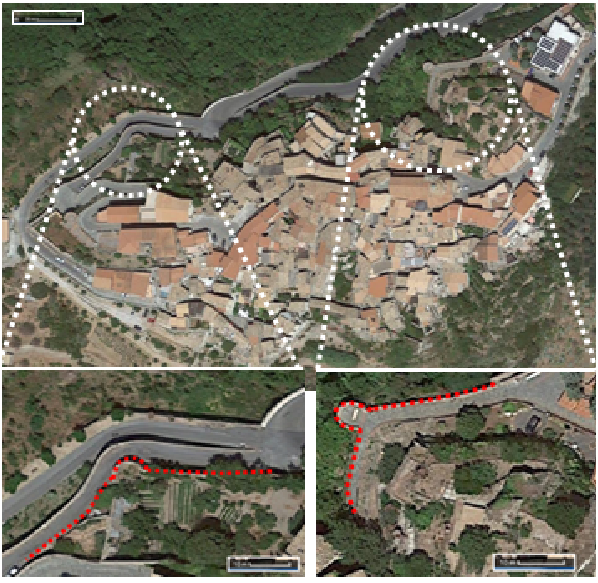

Fig. 4. Il contesto urbano.

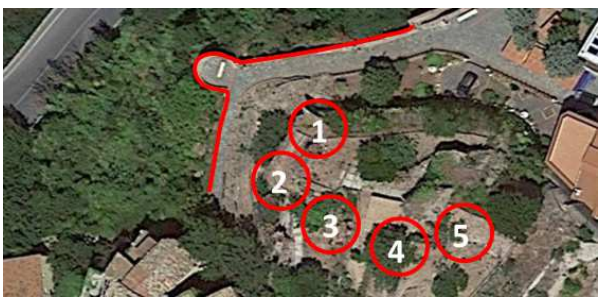

Fig. 5. Zona A: le cinque aree - planimetria.

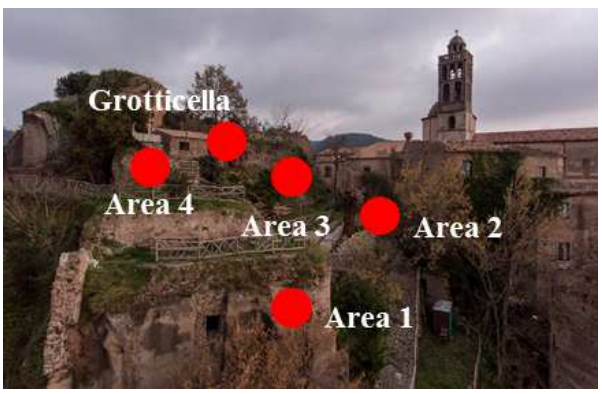

Fig. 6. Zona A: le cinque aree - vista aerea. 

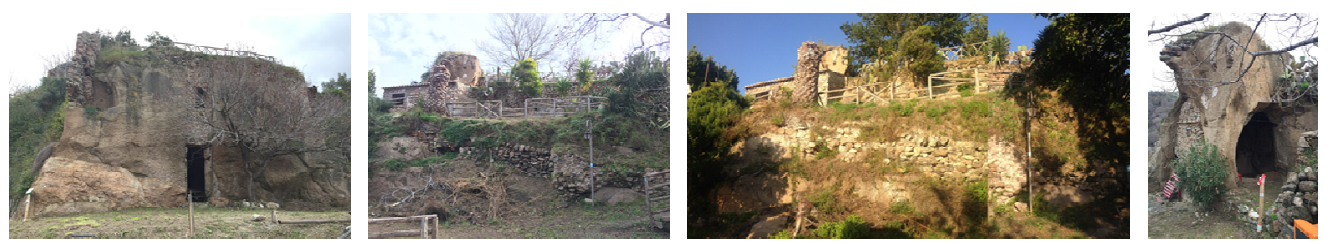

Fig. 7. Scorci delle aree della Zona A.

Nell'area 2 si trova la parte della muratura e della strada in pietra che dava l'accesso al castello, nella 3 vi sono le parti delle mura realizzate con materiali lapidei artificiali.
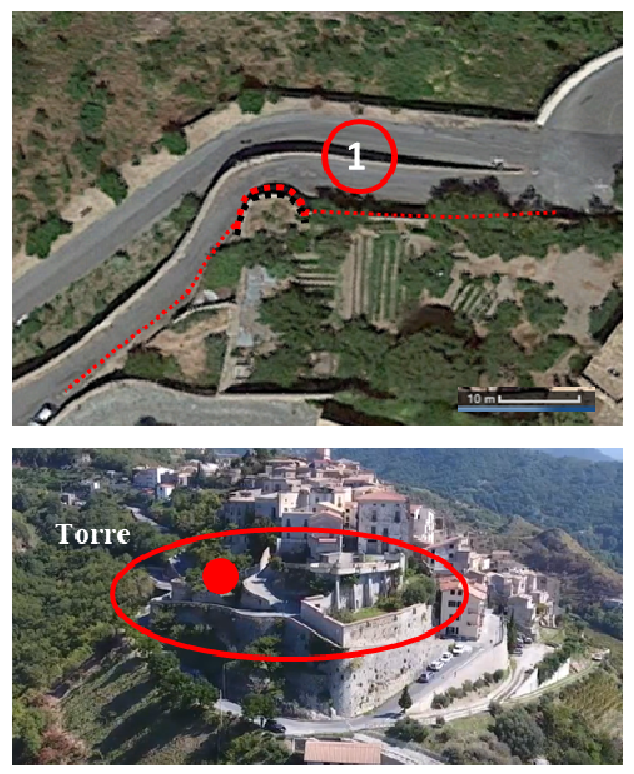

Fig. 8. Zona B: le mura.

Nell'area 4 tra le rovine di ciò che rimane del castello e la parte nuova, si trova una porticina, con un noto portale in legno, risalente al diciottesimo secolo, che dava accesso ad un vano probabilmente usato come dispensa per le provviste durante il lungo periodo invernale. In tale area, la parte in muratura si distingue per forma e aspetto cromatico del tufo e delle pietre locali utilizzati per realizzarla (Matteini, et al., 2003). L'area 5 "grotticella", realizzata con tufo e materiali ricavati in loco e lasciata per molto tempo in stato d'abbandono si presenta oggi in buon stato di conservazione.

La Zona B è composta da due parti, la prima riguarda le mura che delimitavano le residenze dei signori e delle loro famiglie mentre la seconda parte è quella relativa al nucleo storico e al centro delle attività economiche e sede di giustizia. Nello specifico, la prima, parte principale, è costituita dal profilo planimetrico del perimetro murario.

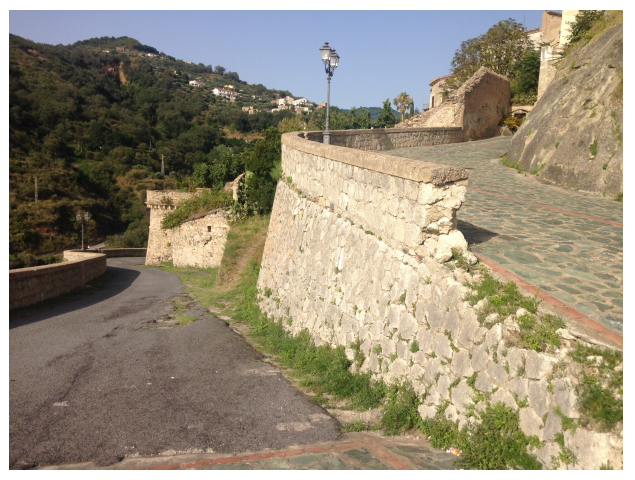

Fig. 9. Un tratto delle mura.

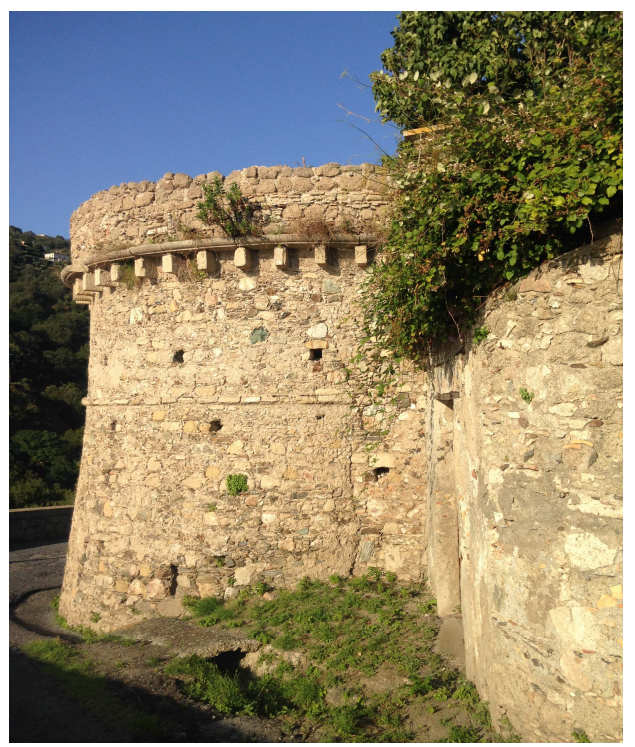

Fig. 10. La torre. 
Esso era in origne scandito da almeno cinque torrioni; quello di maggior rilievo qui oggetto dello studio è il torrione, noto come "Turra", ed è quello che si nota maggiormente data anche la sua posizione paesaggistica (VV.AA., 1993).

Per realizzare le strutture murarie fu utilizzato pietrame squadrato, spianato nei piani di posa e di attesa laterali, messo in opera e collocato in maniera da ottenere serie sovrapposte di corsi il più possibile orizzontali e paralleli.

La torre è composta da due livelli, il primo con un impianto circolare a scarpata è collegato mediante un cordolo marcapiano al livello superiore avente impostazione cilindrica che presenta in sommità una modanatura con decorazione a dentelli.

Essa conserva ancora i caratteri costruttivi e i materiali originari, per tale motivo è apparso necessario in questo studio acquisire ulteriori e più approfondite conoscenze su di essi in quanto è sulla base delle informazioni raccolte ed opportunamente elaborate che diventa possibile impostare e gestire nel modo più corretto un progetto di restauro.

\section{Campionamento e analisi dei materiali}

In ciascuna delle aree esaminate sono stati analizzati e prelevati dei campioni di materiale costituivi che sono stati portati ed analizzati in laboratorio (Accardo, et al., 1989). Per completare la definizione del quadro conoscitivo è stata pertanto svolta una indagine preliminare con l'intento di fornire una linea guida da seguire per analizzare i materiali costitutivi principali (Gattuso, 2014). Sono state quindi svolte delle inda-

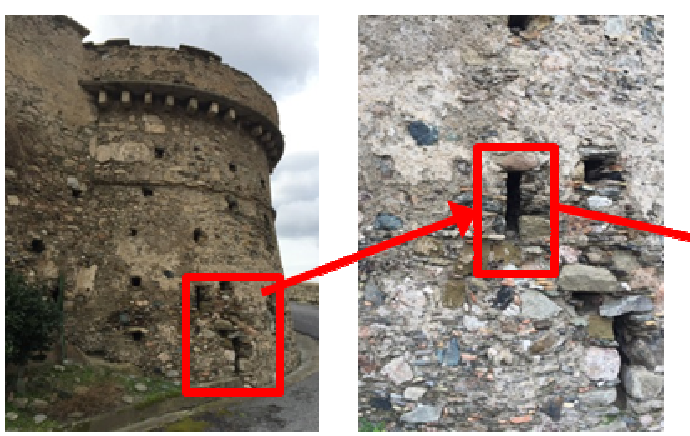

gini sui materiali tenendo in considerazione le parti delle strutture ancora autentiche e quindi i materiali appartenenti alla torre "Turra". A tal fine è stata programmata una campagna di prelievi effettuati durante mirati e specifici e sopralluoghi. Nella fase di campionamento è stato possibile fare un primo censimento dei materiali utilizzati per realizzare le strutture del castello e delle mura. Tale indagine ha permesso di selezionare una tipologia di roccia dal colore scuro individuata come quella usata in maniera più diffusa. A titolo rappresentativo sono qui illustrati i risultati relativi ad un campione prelevato da tale roccia che, portato in laboratorio, è stato sottoposto ad una indagine spettroscopica Raman.

\subsection{Descrizione}

L'analisi riguardante il campione prelevato identificato con la sigla RBC3 (Roccia zona B Castello n.3) è stata eseguita nello specifico utilizzando una strumentazione micro-Raman.

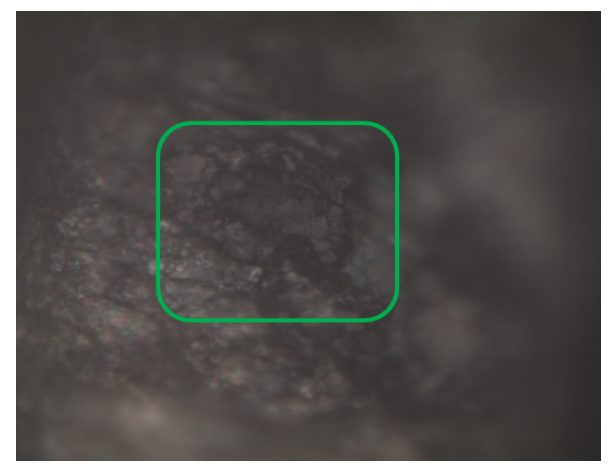

Fig. 11. Immagine al microscopio ottico.

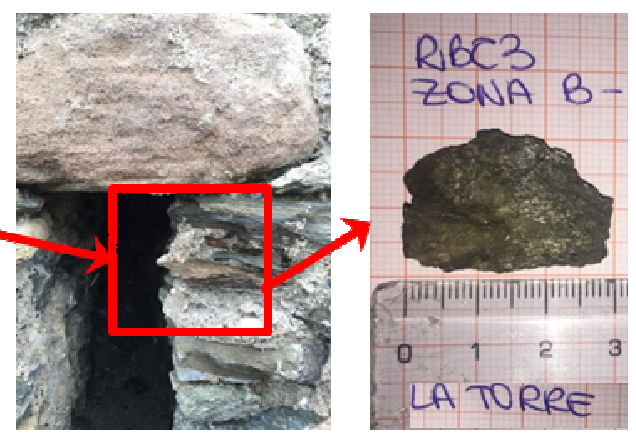

Fig. 12. Le fasi del prelievo del campione RBC3 - Zona B - La torre. 
Il campione prelevato, di colore nero con una dimensione pari a circa $3 \mathrm{~cm}$ di lato, presenta una tessitura piuttosto omogenea.

L'immagine è stata catturata con il microscopio ottico da laboratorio mentre il laser utilizzato è stato il verde (avente $\kappa=532 \mathrm{~nm}$ ).

Il tempo di acquisizione per ciascun punto è stato di circa 20 accumuli ogni 20 secondi.

Le aree sono state determinate dal montaggio degli spettri Raman usando le funzioni Lorentz, prima della rimozione della linea di base.

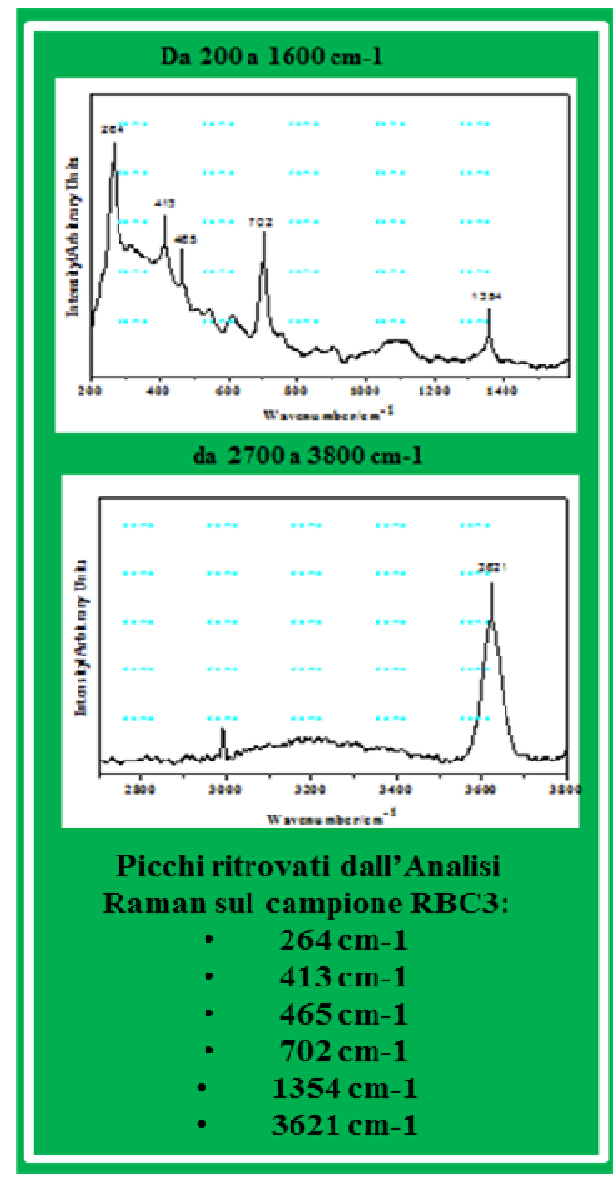

Fig. 13. Spettri Raman.

La spettroscopia Raman consente un rapido riconoscimento delle fasi fibrose, e fornisce delle informazioni sui diversi modi vibrazionali delle molecole (Goldstein, et al., 2018).
Il suo utilizzo ha il vantaggio che:

- Non è necessaria nessuna preparazione specifica dei campioni;

- È una tecnica non-distruttiva;

-È sensibile alle piccole variazioni della struttura chimica.

La spettroscopia Raman è un metodo per osservare transizioni molecolari a bassa frequenza come livelli vibrazionali e rotazionali. Questa tecnica opera sul principio della diffusione anelastica di luce, al contrario della diffusione di Rayleigh che utilizza la dispersione della luce elastica (Gallone, 1989).

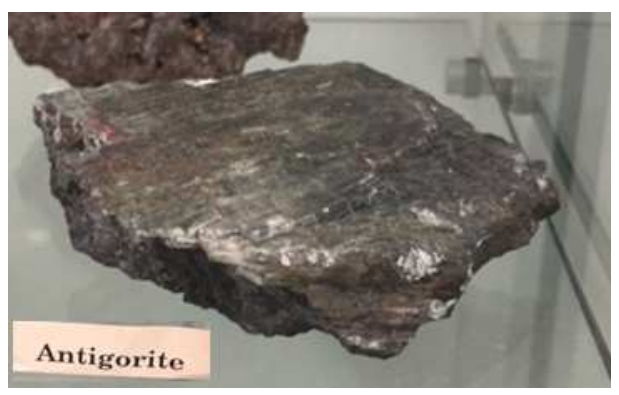

Fig. 14. Campione si Antigorite.

La foto, scattata in laboratorio, mostra un campione di Antigorite utilizzato per effettuare il confronto a occhio con il campione prelevato.

Il minerale che appartiene al gruppo del serpentino, si presenta sempre con abito lamellare (Morbidelli (2003).

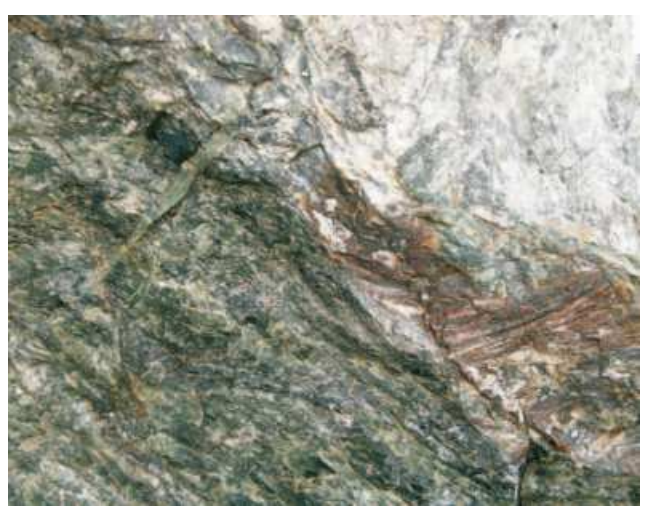

Fig. 15. Abito lamellare dell'antigorite. 
Il campione in esame mostra vene a olivina + antigorite + diopside + Ti-clinohumite + magnetite: si tratta di vene associate all'evento metamorfico in facies eclogitica.

\section{Caratteristiche strutturali principali:}

Colore: verde, blu-verde, bianco, marrone, nero;

Aspetto: vitreo;

Durezza: 3 1/2 -4 scala Mohs / Densità: 2,5 $2,6 \mathrm{~g} / \mathrm{cm} 3$;

Sistema cristallino: monoclino;

Membro di: Serpentine > Kaolinite;

Abito: aggregati di cristalli a strutture lamellare;

Rilievo: assente;

Paragenesi: prodotto di trasformazione di oli-

vine e pirosseni su cui è spesso pseudomorfo.

Tab. 1. Tabella delle principali caratteristiche.

L'antigorite si presenta come un aggregato policristallino in cui le lame antigoritiche hanno dimensioni di diversi decimi di $\mathrm{mm}$. Viene ritenuto monoclino (Wicks, O'Hanley, 1988), si forma durante il metamorfismo, di basso grado, di rocce basiche e ultrabasiche (peridotiti, gabbri, lamprofiri etc.) ad alta temperatura ossia solo sopra i $250^{\circ} \mathrm{C}$.

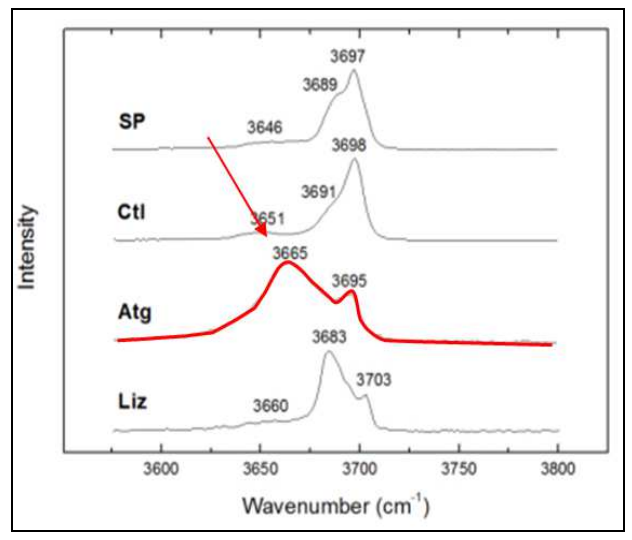

Fig. 16. Spettri dei minerali del gruppo Serpentino.
Nelle rocce ultrabasiche l'antigorite ha spesso una tessitura a griglia; questo è dovuto al fatto che i cristalli crescendo secondo due direzioni preferenziali e intersecandosi tra loro, formano una griglia. Il picco a $3621 \mathrm{~cm}-1$ attribuito al gruppo $\mathrm{OH}$ è confermato in letteratura.

\section{Conclusioni}

Per realizzare un adeguato progetto finalizzato alla definizione di interventi da attuare su un monumento storico-architettonico è necessario adottare una metodologia conoscitiva che consenta di acquisire informazioni in grado di delinearne uno specifico quadro conoscitivo.

Per essere efficace essa deve prevedere il contributo di più competenze a vario livello coinvolti in relazione al livello e al tipo di approfondimento che si vuole raggiungere.

Lo studio svolto tiene conto dei caratteri e dei valori materiali ed immateriali del manufatto architettonico in esame. Il metodo utilizzato ha avuto l'obiettivo di acquisire, ponendosi con atteggiamento flessibile, una conoscenza il più possibile completa che si è adattata e modificata in maniera opportuna lungo il percorso conoscitivo, in relazione alle informazioni che con lo studio si andavano raccogliendo.

In tal modo infatti è stato svolto lo studio del castello di Belmonte che si è soffermato in particolare sulla sua torre la cui presenza fisicomateriale, nel rappresentare il simbolo della cittadina, acquisisce una dimensione culturale connotante e di notevole rilievo.

Dall'analisi del contesto territoriale e urbano la ricerca è proseguita ponendo una particolare attenzione a tutti gli aspetti relativi alle sue varie vicissitudini storiche avvenute nel tempo effettuando nel contempo una sua descrizione architettonica, che ha permesso di individuare le parti originali rimaste intoccate.

L'iter metodologico interdisciplinare utilizzato ha compreso approfondimenti riguardanti i materiali e la struttura della torre. A tal fine è stata effettuata una analisi effettuando, durante una campagna di campionamento, dei prelievi da una parte ancora autentica della torre. 
Nello specifico l'attenzione si è soffermata su uno di essi, selezionato tra tutti quelli prelevati per la sua rappresentatività che è stato analizzato utilizzando la tecnica raman in laboratorio.

Lo studio di un monumento qual è la torre di Belmonte finalizzata alla definizione del quadro preciso delle sue condizioni implica la raccolta delle conoscenze necessarie che vanno attuate in modo interdisciplinare, ritenute indispensabili per poter predisporre un piano di intervento attento e adeguato in grado di fornire indicazioni per garantire la sua corretta conservazione ed anche per predisporre progetti in grado di valorizzare la sua identità che deve essere rispettata in tutti i suoi aspetti.

\section{Bibliography}

Accardo, G.;Vigliano, G. (1989). Strumenti e materiali del restauro. Metodi di analisi, misura e controllo, Kappa Ed., Roma.

Gallone, A. (1989). Analisi fisiche e conservazione, Franco Angeli Ed., Milano.

Gattuso, C. (2011). "Per un approccio razionale al piano diagnostico", in Atti del IIth Convegno Internazionale AIESDiagnosi per la conservazione e valorizzazione del Patrimonio Culturale, Ethos Ed., Napoli.

Gattuso, C. (2012). "A coordinated and multi-step approach for conservation and enhancement of built heritage", in Atti del convegno IIIth Convegno Internazionale AIES- Diagnosi per la conservazione e valorizzazione del Patrimonio Culturale, Ethos Ed., Napoli.

Gattuso, C. (2014). "An Advanced Model to Represent and Manage Knowledge in Cultural Heritage", in XII International Forum Le Vie dei Mercanti - Best pratice in heritage conservation management, La scuola di Pitagora Ed., Napoli.

Goldstein, J.; Goldstein, J.; Newbury, D.E.; Joy, D.C.; Lyman, C.E.; Echlin, P.; Lifshin, E.; Sawyer, L.; Michael, J.R. (2018). Scanning electron microscopy and x-ray microanalysis, Springer Ed., New York.

Matteini, M.; Moles, A. (2003). Science and Restoration - methods of investigation, Nardini Ed., Firenze.

Mordibelli, L. (2003). Le rocce e i loro costituenti, Bardi Ed., Roma.

Valente, G. (1973). Dizionario dei luoghi della Calabria, Frama Sud Ed., Chiaravalle Centrale.

VV.AA. (1993). Per un atlante della Calabria. Territorio, insediamenti storici, manufatti architettonici, Gangemi Ed., Roma. 